\title{
Zur Tätigkeit bei der Direktion für Entwicklungs- zusammenarbeit und humanitäre Hilfe (DEH) ${ }^{11}$
}

\section{Grundsätze und Ziele der Arbeit der DEH}

Dieses Jahr sind es 20 Jahre, daß mit dem Dienst für technische Zusammenarbeit (DftZ), der heutigen Direktion für Entwicklungszusammenarbeit und humanitäre Hilfe $(D E H)$, eine eigene Bundesstelle geschaffen worden ist, die sich mit der internationalen Entwicklungszusammenarbeit befaß $\mathrm{t}^{2}$. Wenn auch manche Theorien, manche Ansichten über das Wie der Entwicklungszusammenarbeit in diesen zwei Jahrzehnten aufgrund der Erfahrungen Änderungen erfahren haben, geblieben ist der Grundsatz dieser Zusammenarbeit, wie er im Artikel 2 des Bundesgesetzes über internationale Entwicklungszusammenarbeit und humanitäre Hilfe (vom 19.3.1976) formuliert ist:

«Die internationale Entwicklungszusammenarbeit und humanitäre Hilfe sind Ausdruck der Solidarität, die eines der Prinzipien darstellt, nach denen die Schweiz ihr Verhältnis zur internationalen Gemeinschaft gestaltet, und entsprechen der weltweiten Verflechtung. Sie beruhen auf der gegenseitigen Achtung der Rechte und Interessen der Partner.

Die Maßnahmen nach diesem Gesetz berücksichtigen die Verhältnisse der Partnerländer und die $\mathrm{Be}$ dürfnisse der Bevölkerung, für die sie bestimmt sind.

Die Leistungen des Bundes erfolgen unentgeltlich oder zu Vorzugsbedingungen. Sie ergänzen in der Regel eigene Anstrengungen der Partner.»

Von diesem Grundsatz leiten sich die Ziele der Entwicklungszusammenarbeit ab, die im gleichen Bundesgesetz (Art.5) folgendermaßen festgehalten sind:

«Die Entwicklungszusammenarbeit unterstützt die Entwicklungsländer im Bestreben, die Lebensbedingungen ihrer Bevölkerung zu verbessern. Sie soll dazu beitragen, daß diese Länder ihre Entwicklung aus eigener Kraft vorantreiben. Langfristig erstrebt sie besser ausgewogene Verhältnisse in der Völkergemeinschaft.

Sie unterstützt in erster Linie die ärmeren Entwicklungsländer, Regionen und Bevölkerungsgruppen. Sie fördert namentlich

a) die Entwicklung ländlicher Gebiete;

b) die Verbesserung der Ernährungslage, insbesondere durch die landwirtschaftliche Produktion zur Selbstversorgung; c) das Handwerk und die örtliche Kleinindustrie;

d) die Schaffung von Arbeitsplätzen;

e) die Herstellung und Wahrung des ökologischen und demografischen Gleichgewichts.»

Diese Ziele sind mit Absicht weit gefaßt, um den Bedürfnissen eines einzelnen Landes, den lokalen Voraussetzungen und Möglichkeiten Rechnung tragen zu können. Um diese weitgespannten Ziele, die für die Arbeit der DEH als Richtschnur gelten, zu erreichen, muß die DEH verschiedenste Mittel einsetzen, je nach den spezifischen Umständen einer Einzelsituation. Nicht nur der organisatorische Ablauf eines Projektes, sondern auch die Wahl des Partners (Ministerien, lokale Verwaltungen, private Organisationen oder internationale Hilfsorganisationen) und die Form der Zusammenarbeit sind den speziellen Gegebenheiten anzupassen.

Es ist daher auch schwierig, ein typisches Projekt ${ }^{3)}$ der DEH vorzustellen, um daran die Arbeit der für die Projekte zuständigen Mitarbeiter ( «Sachbearbeiter») zu zeigen.

\section{Beispiel eines Projektablaufs}

Das Projekt ${ }^{4}$ ), welches im folgenden in seinem chronologischen und organisatorischen Ablauf dargestellt werden soll (siehe Fig. 1), ist insofern typisch, als sich hier in den verschiedenen Phasen der Projektidentifikation, der Projektvorbereitung und der Projektdurchführung zahlreiche Beziehungen, Maßnahmen und Entscheidungen vorfinden, die in einer Großzahl der DEH-Projekte ebenfalls in der einen oder anderen Form enthalten sind. Da es sich um die Realisierung eines einfachen und "greifbaren» Projektzieles handelt, hat das Projekt den Vorteil der Übersichtlichkeit. Dagegen illustriert es einen eher atypischen Tätigkeitsbereich. Als isoliertes Projekt ist das hier dargestellte Beispiel außerdem für Formen einer kontinuierlichen Zusammenarbeit mit bestimmten Partnern, wie sie sich in zahlreichen Schwerpunktländern einspielen und aus welchen oft verschiedene, parallel laufende oder aufeinanderfol-

Dr. phil. nat. Hermann Escher, Direktion für Entwicklungszusammenarbeit und humanitäre Hilfe. Bern. 


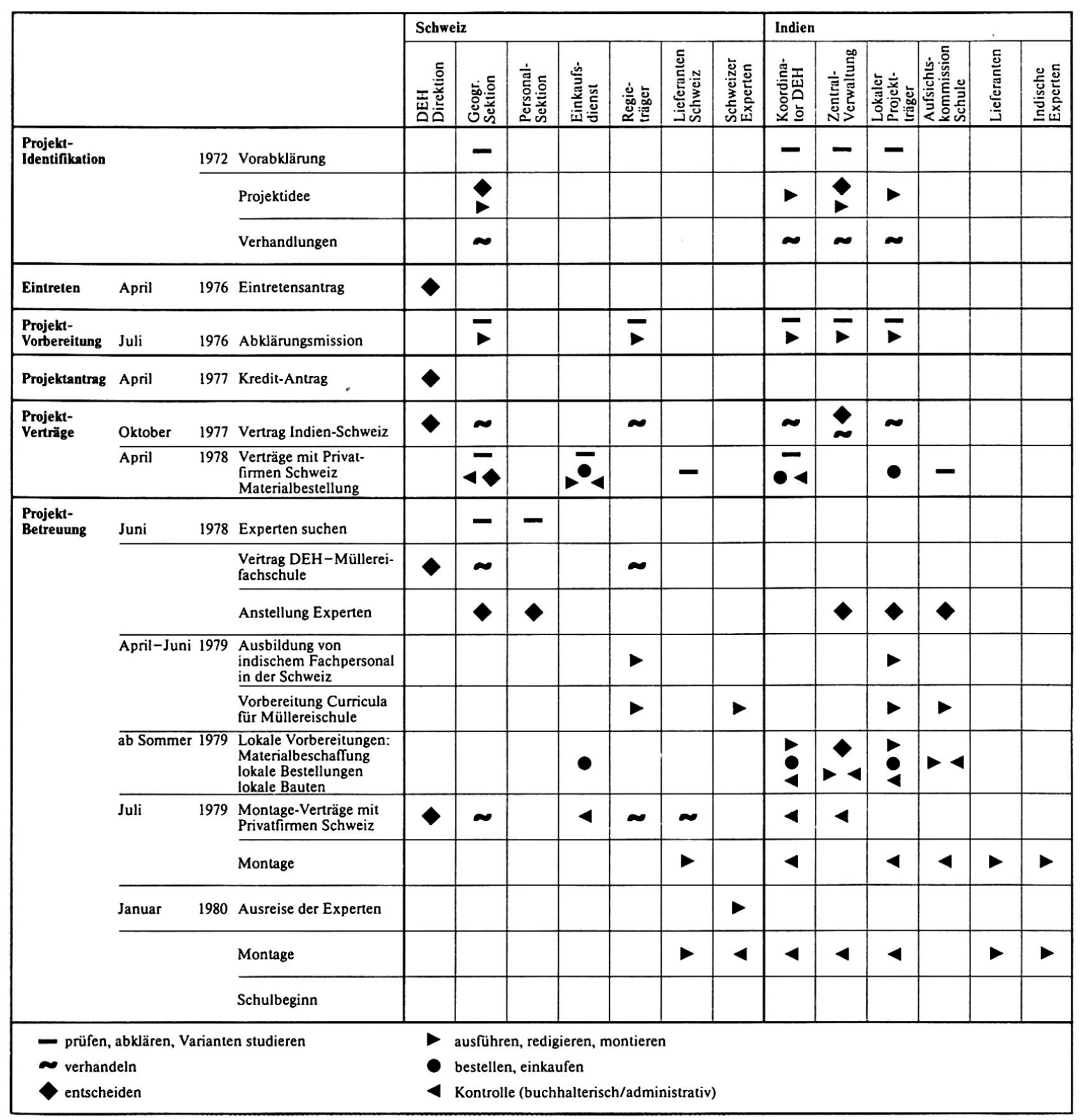

Fig. 1: Schematische Darstellung des Ablaufs des Projektes \&Internationale Müllereifachschule in Mysore)

gende Projekte hervorgehen, weniger charakteristisch.

\subsection{Das Projekt}

Beispiel: Internationale Müllereifachschule in Mysore (Indien).

Projektziel: Aufbau einer internationalen Müllereifachschule in Mysore zur Ausbildung von jährlich 20 Müllerei-Technikern.

Leistungen der Schweiz: Lieferung der Laborausrüstung, Erstellung einer Schulmühle, technische und didaktische Beratung, Mitwirkung bei der Ausbildung des indischen Lehrkörpers.

Bewilligter Kredit: 1,5 Millionen Franken.

\subsection{Zur Projektidentifikation}

Die Idee einer schweizerischen Beteiligung am Aufbau einer Müllereifachschule in Indien wurde 1972 durch den indischen Müllereiverband an den Koordinator der DEH in New Delhi herangetragen.

Der Koordinator ist als Vertreter der DEH Vermittler zwischen den schweizerischen Projekten in sei- 
nem Tätigkeitsgebiet und der Zentrale in Bern. Vor allem aber vertritt er die DEH gegenüber der Verwaltung des betreffenden Landes, ist offen für Initiativen und Projektideen und bemüht um eine realistische Darstellung der Möglichkeiten und Spezialisierungen der schweizerischen Entwicklungszusammenarbeit.

Es existieren in der westlichen Welt nur zwei Müllereifachschulen: die eine befindet sich in Kansas City, die andere in St. Gallen. In der Schweiz hat auch einer der größten internationalen Lieferanten von $\mathrm{Zu}$ behör für das Müllereigewerbe seinen Sitz. Es war daher naheliegend, daß sich der Müllereiverband in diesem Fall mit einer Anfrage an die Schweiz wandte. Bevor der Koordinator die Anfrage an die Zentrale in Bern weiterleitete, nahm er in New Delhi weitere Abklärungen vor.

Gute Kontakte im Land und ein hoher allgemeiner Informationsstand sollen es dem Koordinator erlauben, sich bei Anfragen ein Bild über deren Hintergrund verschaffen zu können. Bei der Vorprüfung einer ersten Projektidee gilt es vor allem abzuklären, auf welche Grundannahmen und Bedürfnisse das Projekt aufbaut, wie sich das Projekt in andere Entwicklungsvorhaben und in die Entwicklungspläne der Regierung einfügt und von welchen Kreisen es unterstützt wird.

Die Abklärungen ergaben folgendes Bild: In Indien waren zu jenem Zeitpunkt 260 industrielle Mühlen in Betrieb, die infolge eines krassen Mangels an ausgebildetem Personal (insgesamt 15 in St. Gallen oder in Kansas City ausgebildete Techniker) oft unsachgemäß betrieben wurden. Dies führte zu mangelhaftem technischen Unterhalt der Mühlen, zu schlechter Mehlqualität und zu unnötig hohen Verlusten.

Die indische Regierung unterstützte die Bestrebungen, eine Müllereifachschule in Indien zu errichten. Während der letzten zehn Jahre war Indien durch die Einführung ertragreicher Sorten, durch die Ausdehnung der Anbauflächen und durch andere Maßnahmen zum drittgrößten Getreideproduzenten der Welt geworden. Die Regierung erhoffte durch eine maßgebliche Erhöhung gut ausgebildeter Müllereifachleute eine Reduktion der Verluste bei der industriellen Müllerei und damit eine weitere indirekte Ertragssteigerung.

Nach Übermittlung einer Anfrage und zusätzlicher Information durch den Koordinator findet auch in Bern jeweils eine erste Abklärung statt.

Bezüglich der Anfrage des indischen Müllereiverbandes waren insbesondere folgende Fragen genauer abzuklären:

- Entspricht dieses Anliegen den Zielsetzungen, die mit der schweizerischen Entwicklungszusammenarbeit angestrebt werden?

- Wäre ein solches Projekt mit den Finanzplänen und personellen Kapazitäten der DEH vereinbar?

- Ist es technisch durchführbar?

\subsection{Zum Eintreten}

Führen die ersten Abklärungen nicht zu einem negativen Ergebnis, formuliert die zuständige geographische Sektion (das heißt vor allem der Sachbearbeiter) in Bern, gestützt auf die Informationen des Koordinators, einen Eintretensantrag an die Direktion. Bei positivem Eintretensentscheid verfügen Sachbearbeiter und Koordinator über die Rückendeckung zur Aufnahme konkreter Verhandlungen mit Projektpartnern im betreffenden Entwicklungsland und in der Schweiz.

Der lange Zeitraum zwischen Projektidee und Eintretensentscheid erklärt sich durch eine Verzögerung durch eine parlamentarische Anfrage in Bern, welche die schweizerische Entwicklungshilfe in Indien wegen der dort vorgenommenen Atomversuche in Frage stellte.

Bei der Eintretensdebatte zur Müllereifachschule gab vor allem die Frage Anlaß zur Diskussion, ob ein solches Projekt zur Unterstützung der industriellen Müllerei nicht den mit Entwicklungszusammenarbeit der DEH verfolgten Zielen widerspreche. In Indien wird das Mehl für den Hausgebrauch entweder in den Haushalten durch Zermalmen zwischen zwei Steinen oder in kleinen, mit Diesel- und Elektromotoren betriebenen Mühlen hergestellt. Die Lagerung des Korns, das erst bei Bedarf gemahlen wird, in den Haushalten ist vorteilhaft. Die rund 100000 Kleinmühlen auf dem Land und rund 40000 Kleinmühlen in der Stadt, die in der Regel von zwei Personen betrieben werden, schaffen zahlreiche Arbeitsplätze. Wohl ist ihre Arbeitsweise nicht sehr ökonomisch und das produzierte Mehl nicht immer von erstklassiger Qualität, hier schafft jedoch oft die Konkurrenzsituation von selbst gewisse Korrekturen. Besteht also die Gefahr, so war zu fragen, daß diese dezentralisierte Kleinmüllerei durch die industrialisierte Müllerei verdrängt würde?

Nach Ansicht des Koordinators war dies nicht der Fall: Die industrielle Müllerei produziert in erster Linie für die Bedürfnisse des Bäckereigewerbes und der Biskuitindustrie und konkurrenziert die Kleinmüllerei nur wenig. Die Getreideverluste bei der nicht-industriellen Verarbeitung sind immens, und die Forderung nach nicht-industrieller Verarbeitung der gesamten Getreideernten wäre nicht nur unrealistisch, sondern auch unproduktiv.

Der Eintretensentscheid war schließlich positiv, jedoch mit der Auflage, den aufgeworfenen Fragen bei der weiteren Verfolgung besondere Beachtung zu schenken.

\subsection{Zur Projektvorbereitung}

In Indien klärte der Koordinator nun ab, auf welchen bereits vorhandenen Dienstleistungen das Projekt aufbauen könnte, zu welchen Eigenleistungen Indien bereit ist und wo sich die spezifischen Eng- 
pässe befinden, die mit Unterstützung der Schweiz sinnvoll beseitigt werden können. Es entsprach dabei sowohl dem auf indischer Seite bestehenden Bedürfnis nach größtmöglicher Unabhängigkeit wie auch dem Bedürfnis auf seiten der Schweiz nach ökonomischem Einsatz der Mittel, daß nur dort Materialbeschaffung aus dem Ausland, Zuzug ausländischer Experten oder Inanspruchnahme ausländischer finanzieller Mittel ins Auge gefaßt werden sollte, wo sich dies für die Realisierung des Projekts als unumgänglich erwies.

Für eine dreiwöchige Abklärungsmission konnten der gerade pensionierte Direktor der Müllereifachschule St. Gallen und ein ehemaliger Schüler dieser Schule, der auch als Ökonom ausgebildet war und über Erfahrung in Entwicklungsländern verfügte, gewonnen werden.

Der Bericht der Abklärungsmission wurde in Bern und in Indien - zwischen dem Koordinator, der Verwaltung, dem Müllereiverband und dem Forschungsinstitut für Lebensmitteltechnologie, welchem die Müllereischule angegliedert werden sollte - ausführlich diskutiert. Es mußte auch abgeklärt werden, ob auf seiten Indiens die nötigen Betriebsmittel für die Schule bereitgestellt werden konnten. Zusammen mit den beiden schweizerischen Beratern, die an der Abklärungsmission teilgenommen hatten, wurden ein Zeit- und Vorgehensplan errichtet und eine Materialliste erstellt.

\subsection{Zum Kreditantrag}

Der Kreditantrag ist ein ausführliches, von der zuständigen Sektion (d.h. wiederum vor allem vom Sachbearbeiter) und vom Koordinator verfaßtes Dokument, welches das vorgesehene Projekt in seinem organisatorischen Ablauf darstellt und die Mittel nennt, die dazu von seiten des Entwicklungslandes, der Schweiz und allenfalls weiterer Geldgeber zur Verfügung gestellt werden sollen. Das Projekt wird auch in einen Zusammenhang zur Entwicklungspolitik des betreffenden Landes gesetzt und in seiner allgemeinen Zielsetzung erörtert.

Gestützt auf den Kreditantrag und auf Antrag der Direktion faßte der Vorsteher des Eidgenössischen Departementes für auswärtige Angelegenheiten die Entscheidung, zur Schaffung einer internationalen Müllereischule in Mysore Fr. 1500000.- zur Verfügung zu stellen. Das Projekt sollte der Müllereischule St. Gallen in Regie übergeben werden und auf indischer Seite dem Council of Scientific and Industrial Research unterstellt werden.

\subsection{Zur Projektbetreuung}

In den Verhandlungen des Koordinators in Indien über die konkrete Abwicklung des Projekts spielte die Frage, welche Materialien in Indien beschafft werden sollen und für welche Bestandteile ein Im- port aus dem Ausland unumgänglich war, eine wesentliche Rolle. Für $15 \%$ des gesamten Materialbedarfs wurden schließlich Importlizenzen beantragt. Solche Anträge zu stellen beansprucht sehr viel Zeit, da in jedem Fall der Nachweis erbracht werden muß, daß die betreffenden Artikel in Indien noch nicht hergestellt werden können.

Die Zentrale in Bern trat in Verhandlungen mit dem Lieferanten des zu importierenden Materials. Der Lieferant mußte dafür gewonnen werden, an der konkreten Planung der Schulmühle und des Labors mitzuwirken und seine Pläne indischen Firmen zur Verfügung zu stellen, welche in der Lage waren, die Einrichtungen nach diesen Angaben zu montieren. Die Rekrutierung eines schweizerischen Experten für das Müllereiprojekt erwies sich als äußerst schwierig. Die Löhne schweizerischer Entwicklungsexperten liegen unterhalb der Ansätze im Müllereigewerbe. In dieser Berufssparte sind außerdem Fremdsprachenkenntnisse nicht sehr wichtig, während gute Englischkenntnisse für die Arbeit der Experten in Indien unumgänglich waren.

Für die Projektbegleitung konnte die Müllereifachschule in St. Gallen gewonnen werden.

Die Projekte der DEH setzen vielfach Branchenund Fachkenntnisse voraus, über welche die Sachbearbeiter nicht verfügen. In solchen Fällen wird in der Schweiz ein Projektbegleiter gesucht - oft ein Universitätsinstitut - welcher die Abwicklung des Projekts zusammen mit der zuständigen geographischen Sektion der DEH mitbetreut.

Ein schweizerischer Experte befindet sich seit Beginn dieses Jahres in Mysore. Die Montage der Mühle geht planmäßig voran, mit der Eröffnung der Schule kann im Laufe des Jahres 1981 gerechnet werden.

\section{Die Arbeit des Sachbearbeiters}

Um die zur Verfügung stehenden Mittel mit dem der DEH zugestandenen Personal rationell einsetzen zu können, wird trotz den weit gefächerten Zielen eine Konzentration auf bestimmte Sektoren angestrebt:

Auszahlungen 1979:

Landwirtschaft $\quad 26 \%$

Industrie $\quad 15 \%$

Gesundheit $\quad 14 \%$

Erziehung $\quad 13 \%$

Multisektoriell $\quad 10 \%$

Bauten, Transporte, Handel $9 \%$ nicht klassiert $\quad 13 \%$

Aus gleichen Überlegungen drängt sich auch eine Beschränkung in der Zahl der Länder, mit denen die DEH zusammenarbeitet, auf ( «Schwerpunktländer») $)^{5}$.

Trotzdem ist das Spektrum der Probleme, die der 
Sachbearbeiter zu behandeln hat, sehr vielfältig, wie dies auch schon aus dem oben dargestellten Beispiel hervorgeht. Mit Ausnahme für die Sektoren Landwirtschaft/Forstwirtschaft/soziale Fragen bestehen innerhalb der DEH keine Stellen fachspezifischer Art. Der Sachbearbeiter ist daher darauf angewiesen, auf verschiedensten Gebieten über Grundkenntnisse zu verfügen und zusätzlich mit externen Beratern zusammenzuarbeiten.

Diese Situation macht auch verständlich, daß die bei der DEH tätigen Sachbearbeiter ganz verschiedene Ausbildungen und Erfahrungen mitbringen: Ökonomie, Soziologie, Ingenieurwissenschaften, Geschichte, Recht, Land- und Forstwirtschaft u.a. Unter den Sachbearbeitern sind gegenwärtig drei Geographen, resp. Geographinnen tätig (je eine[r] mit Abschluß in Genf, Bern und Zürich). Alle diese Leute arbeiten vor allem als Generalisten und nur beschränkt auf ihrem eigenem Fachgebiet. Es ist hier anzufügen, daß die DEH nicht nach Fachgebieten (wie z.B. die deutsche Gesellschaft für technische Zusammenarbeit, GTZ), sondern geographisch gegliedert ist, was wiederum - zumindest beim jetzigen Projektvolumen - einen Einsatz der Mitarbeiter nach ihren Fachspezialitäten ausschließt (mit Ausnahme der oben genannten drei Bereiche). Das Beispiel der drei Geographen soll diese Situation verdeutlichen: Zwei Projekte auf dem Gebiet der Fernerkundung, eines im Bereich Geologie werden z. Z. von der DEH finanziert, dazu kommen Projekte mit ökologischen Komponenten: Gesamthaft ein zu kleines Volumen, um einen Geographen ausschließlich damit zu beschäftigen.

Mit Absicht ist bis jetzt nicht die Rede gewesen von dem, was nach landläufiger Meinung die hauptsächliche Arbeit eines Bundesangestellten ausmacht: Administration und Bürokratie. Jeder frischgebackene Universitätabsolvent vermag nur mit Schaudern daran zu denken, daß ihn solche Dinge vom wissenschaftlichen Arbeiten abhalten könnten, einige haben daher auf eine Anstellung beim Bund verzichtet, häufig sicher zu unrecht. $\mathrm{Da}$ es auch bei der DEH nicht ganz ohne Administration geht, liegt auf der Hand. Das Wissen und die Erfahrung, wie die positiven Seiten der Administration für die eigene Arbeit möglichst nützlich eingesetzt werden, eignet man sich indessen bald an. Und gerade hier liegt eine wichtige Aufgabe des Sachbearbeiters: Wie das oben angeführte Projektbeispiel zeigt, sind für das gute Gelingen eines Projekts zahlreiche Schritte, auch solche administrativer Art, nötig. Werden diese möglichst effizient und für das Projekt nutzbringend gestaltet, können sie sich nur positiv auswirken, genauso wie das Gegenteil ein Projekt fast völlig zu lähmen vermag.

Eine eigentliche Ausbildung für die Arbeit des Sachbearbeiters besteht nicht. Wichtig ist einerseits eine breite Grundausbildung (inkl.Sprachkenntnisse) sowie die Bereitschaft zur Zusammenarbeit mit anderen Disziplinen. Ebenso bedeutend sind aber andererseits Toleranz und Verständnis für andere Kulturen und Denkweisen, für soziale Probleme und Zusammenhänge sowie der Wille, sich ernsthaft damit auseinanderzusetzen. Sinnvolle Entwicklungszusammenarbeit bedeutet Kulturbegegnung, ruft also geradezu nach einer Betrachtungsweise, die über eine rein technische hinausgeht.

Eine Grundlage für die Arbeit des Sachbearbeiters können der Nachdiplomkurs an der ETH Zürich («Nachdiplomstudium für Entwicklungsländer») und die Kurse des «Institut universitaire d'Etudes du Développement» (IUED) in Genf geben. Auch als Vorbereitung auf einen Experteneinsatz sind diese Kurse nur zu empfehlen.

Anders als die Arbeit des Sachbearbeiters an der DEH-Zentrale in Bern ist die Tätigkeit der Experten im Feld gelagert. Sie sind diejenigen, die in Zusammenarbeit mit einheimischen Kollegen die jeweiligen Projekte realisieren. Unter den gegenwärtig ca. 350 Feldmitarbeitern der DEH befinden sich fünf Geographen (dazu noch einer in einem multilateralen Projekt). Sie befassen sich mit Fragen der Grunddatenerhebungen, die für die Planung in den betreffenden Ländern von Wichtigkeit sind, wobei vor allem mit Luft- und Satellitenbildern gearbeitet wird. Hier kommt - viel mehr als in der Tätigkeit des Sachbearbeiters - die eigentliche geographische Arbeit zum Zuge ${ }^{6}$.

Neben den operationellen Aufgaben sind vom Sachbearbeiter auch Fragen der Konzeption und Probleme grundsätzlicher Art der Entwicklungszusammenarbeit zu behandeln. Es geht dabei weniger um streng wissenschaftlich fundierte theoretische $\mathrm{Be}$ trachtungen als um eine Verbindung von Theorie und Praxis, die wieder in die tägliche Projektarbeit einfließen soll.

Bei allen Arbeiten kommen auch immer wieder politische Probleme zur Diskussion, da ja die DEH, als Teil des Eidgenössischen Departements für auswärtige Angelegenheiten (EDA), ihre Arbeit in den allgemeinen Rahmen der schweizerischen Außenpolitik zu stellen hat. Sie steht aber auch im innenpolitischen Spannungsfeld, gibt doch das Für und Wider die Entwicklungszusammenarbeit in der Öffentlichkeit und im Parlament immer wieder zu Diskussionen Anlaß. Damit ergeben sich Rahmenbedingungen, die neben den reinen Projektaspekten zu berücksichtigen sind, ja manchmal sogar im Widerspruch zu diesen stehen.

Trotzdem für ein Projekt gangbare Lösungen zu finden, gehört mit zu den interessantesten Aufgaben des Sachbearbeiters. Als zusätzliche Rahmenbedingungen ist natürlich den Gegebenheiten des Partnerlandes ebenso sehr Rechnung zu tragen.

Aus dem bisher Gesagten läßt sich folgende Bilanz über die Arbeit des Sachbearbeiters ziehen: Die 
fachspezifische Tätigkeit steht weit im Hintergrund. Das ist bei der heutigen DEH-Struktur wohl kaum zu ändern, birgt aber eine gewisse Gefahr in sich, ohne die nötigen Fachkenntnisse Entscheide fällen zu müssen. Dieser zu begegnen ist nur mit einer breiten Ausbildung und - wo nötig - spezifischer Fachberatung möglich.

Ein zweiter Punkt: Das Redigieren von Briefen, Aktennotizen und Projektanträgen gehört zum täglichen Brot des Sachbearbeiters, ebenso Diskussionen über ein Projekt oder allgemeine Fragen der Entwicklungszusammenarbeit in größerem Kreis. Eine

1) Ich danke meinen Kollegen, H. Moos und K. Vögele, für ihre Anregungen und Kommentare.

2) Die DEH ist Teil des Eidgenössischen Departements für auswärtige Angelegenheiten (EDA)

3) Unter einem "Projekt» wird hier der vertraglich festgelegte, konzeptionelle und administrative Rahmen verstanden, innerhalb welchem die DEH in einem bestimmten Land zusammen mit lokalen und nationalen Partnern auf ein konkretes Ziel hinarbeitet.

4) Die nachfolgende Beschreibung und Fig. 1 sind mit geringen Änderungen dem «E + D», Nr.6 (Bern, 1980, herausgegeben gewisse Freude daran sowie Gewandtheit in schriftlichem und mündlichem Ausdruck erleichtert vieles. Ein Drittes und Letztes: Die Tätigkeit des Sachbearbeiters ist vielseitig und abwechslungsreich, sie stellt aber auch hohe Anforderungen an Urteilsvermögen, Erfassen von Zusammenhängen, Einsetzen von Grundlagenkenntnissen und Erfahrungen Dritter zur speditiven Lösung anstehender Probleme. Dem Abwägen des politisch-technisch Machbaren gegenüber dem vom Projektauftrag Wünschbaren kommt häufig entscheidende Bedeutung zu. von der $\mathrm{DEH}$ ) entnommen. Der Text im «E + D» ist vom Autor dieses Artikels maßgeblich mitgestaltet worden.

5) Liste der Schwerpunktländer nach Kontinenten:

Afrika: Sahel (Mali, Niger, Obervolta, Tschad), Madagaskar, Rwanda, Tansania, Kamerun.

Lateinamerika: Peru, Bolivien, Paraguay.

Asien: Bangladesh, Indien, Nepal, Pakistan

6) Vergleiche dazu die Antrittsvorlesung von PD Dr. K. Itten, Universität Zürich, 3.6.1980.

\section{Literaturbesprechung}

NOIN Daniel: Geographie de la population.

328 S., 87 Abb., Masson, Paris, 1979.

wOODS Robert: Population analysis in geography. 278 S., 80 Abb., 60 Tab., Longmann London, 1979 , \& 6.50 .

Der Fachbereich der Bevölkerungsgeographie hat in den letzten zwanzig Jahren viele neue Impulse erhalten. Durch die Möglichkeit der elektronischen Datenverarbeitung und des ständig verbesserten statistischen Datenmaterials entstanden unzählige Studien über Bevölkerungsgruppen verschiedenster Teilgebiete. Viel seltener sind grossräumige Untersuchungen und Arbeiten, die einen Ueberblick über das ganze Spektrum der Thematik geben.

D. NOIN hat durch das vorliegende Werk ein Lehrbuch geschaffen, das dem Studenten die gesamte Problematik der Bevölkerungsgeographie aufzeigt. Auch kulturelle, ethnische, wirtschaftliche und geschichtliche Aspekte werden berücksichtigt. Als wichtigstem Faktor im kulturräumlichen Gefüge hat der Autor einen Schwerpunkt auf die räumliche Verteilung des Menschen und die Disparitäten verschiedener Bevölkerungsgruppen gelegt, etwas kürzer ist der grosse Problemkreis der Mobilität gehalten. Besonders wertvoll sind die vielen Abbildungen, welche dem Studenten die Verarbeitung des Stoffes sehr erleichtern für ihn ist es eine ideale Einführung in die
Bevölkerungsgeographie. Dem Forscher, der etwas tiefer in gewisse Probleme eindringen möchte, sind die vielen Literaturangaben nach jedem Kapitel willkommen. Die aufgeführten Werke sind zum grössten Teil sehr aktuell, die meisten wurden nach 1970 publiziert. Schade, dass gerade die Arbeiten deutscher Sprache sehr unsorgfältig bibliographiert wurden (z.B. 'Witthauer K.: Ungleichmässige Verteilung von 4 Milliarden Menschen, ...").

Völlig anders in seiner Art ist das Lehrbuch von R. WOODS. Das Hauptgewicht liegt in der sorgfältigen Behandlung und Erklärung der statistisch-demographischen Methoden, mit denen Probleme der Bevölkerungsgeographie angegangen werden können. Im Gegensatz zum oben besprochenen Buch wird hier auf eine kulturelle oder ethnische Betrachtungsweise verzichtet. Die Dynamik der Bevölkerungsentwicklung in Zeit und Raum wird zur Wertematrix abstrahiert und mathematisch präzis durchleuchtet.Für Analysen (oder Prognosen) von Fruchtbarkeit, Mortalität oder Migration werden eine Fülle Kennziffern definiert, an Beispielen deren Berechnung erläutert. Wenn dem Studenten im Einzelfall auch die nötigen demographischen Grunddaten für eine bestinmte Region fehlen sollten, so ist dieses Buch als Arbeitshilfe generell sehr empfehlenswert.
GH $2 / 81$
Guido Dorigo, Zürich 